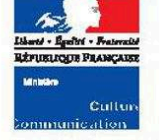

Secrétariat général

Délégation

au développement

et aux affaires

internationales

Département

des études,

de la prospective
et des statistiques

182, rue Saint-Honoré, 75033 Paris cedex 01

중 0140157913 - 图 0140157999

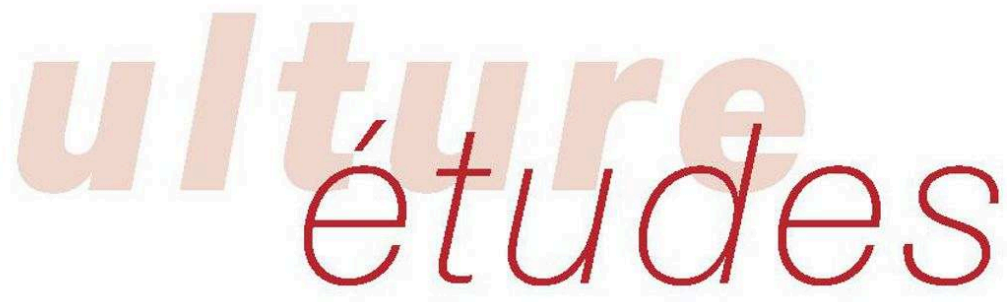

\title{
Image de soi-image du visiteur et pratiques des musées d'art
}

\section{Hana Gottesdiener, Jean-Christophe Vilatte, Pierre Vrignaud}

\section{Self-image/image of the gallery-goer, and art gallery practice}

\begin{abstract}
Avant-propos
Au moment où les établissements culturels comme tous les responsables de politiques de publics s'interrogent sur les conditions de relance des objectifs de démocratisation culturelle, il est fécond d'analyser les frontières qui dépassent, sans les contester, les puissants facteurs sociodémographiques des pratiques culturelles. La recherche présentée ici trouve sa place dans cette démarche. Son apport tient à la nouveauté de son analyse sur «l'image du visiteur », mais surtout à la comparaison qu'elle établit avec «l'image de soi » qui permet de rendre compte des pratiques muséales, notamment en ce qui concerne les musées d'art. La proximité ou la distance entre ces deux images sont en effet de nature non seulement à permettre de dégager des éléments prédictifs de la fréquentation, mais également à fournir un cadre d'interprétation plus général des comportements en matière de pratiques et/ou consommations culturelles légitimes. On constate une nouvelle fois que la médiation occupe une place centrale comme facteur de démocratisation culturelle, mais sur des perspectives renouvelées.

Philippe Chantepie
\end{abstract}

Après avoir montré l'importance des facteurs sociodémographiques dans la fréquentation des institutions culturelles, les études menées sur les publics ont révélé celle des facteurs environnementaux, liés notamment aux paramètres de l'offre et aux modalités de fréquentation. Ce qui fait l'identité d'un individu et le poids de cette identité dans la définition des conduites sociales et dans leur régulation joue également un rôle central dans la fréquentation des musées. Si les liens entre cette fréquentation et les représentations ou l'image que les visiteurs se font des musées, de leurs collections, des outils de médiation ont fait l'objet de nombreuses études ${ }^{1}$, en revanche la question de l'image ou de la représentation de ce qu'est un visiteur de musée ne semble pas avoir été abordée ${ }^{2}$, peut-être parce que ces deux thématiques - image de soi et fréquentation sont traditionnellement traitées séparément, la première par la psychologie, la seconde, le plus souvent, par la sociologie descriptive ${ }^{3}$. Or, c'est une interrogation à envisager si l'on veut comprendre les ressorts des pratiques culturelles en général et de la visite des musées d'art en particulier.

\footnotetext{
* Hana GotTesdiener est professeure émérite de psychologie à l'université Paris X-Nanterre, chercheuse au Laboratoire culture et communication de l'université d'Avignon et des Pays du Vaucluse, directrice et corédactrice en chef de la revue Culture \& Musées. Jean-Christophe VILATTE est mâtre de conférences en sciences de l'éducation à l'université Nancy 2 et chercheur au Laboratoire culture et communication de l'université d'Avignon et des Pays du Vaucluse. Pierre VRIGnaud est professeur de psychologie à l'université de Paris X-Nanterre et chercheur au laboratoire Processus cognitifs et conduites interactives de la même université. Cette étude a été suivie par Sylvie Octobre au DePS. 1. David AliBerT, Régis Bigot et Georges HatCHUEL, Fréquentation et image des musées en début 2005, Paris, CreDoc, coll. "Rapports" 1. David ALIBERT, Regis BIGOT et Georges HATCHUEL, Frequentation et image des musées
$\left(\mathrm{n}^{\circ} \mathrm{R} 240\right.$ ), juin 2005 (document réalisé à la demande de la Direction des musées de France).

2. À partir de la consultation des principales bases de données en sciences humaines et sociales, il n'a pas été possible d'identifier des études 2. A partir de la consultation des principales bases de donnees en sciences humaines et sociales, il n'a pas eté possible d'identifier des études
sur les représentations du visiteur de musée, les mots clés «représentation» ou «image» renvoyant à des travaux sur les représentations du lieu muséal et de ses contenus.

3. L'interrogation des principales bases de données en sciences humaines et sociales en croisant ces deux thematiques n' a pas permis de mettre en évidence l'existence de travaux traitant de cette relation.
} 


\section{Image de soi-image du visiteur et pratiques des musées d'art}

Self-image/image of the gallery-goer, and art gallery practice

Hana Gottesdiener, Jean-Christophe Vilatte et Pierre Vrignaud

Éditeur : Département des études, de la prospective et des statistiques

Lieu d'édition : Paris

Année d'édition : 2008

Date de mise en ligne : 8 juillet 2015

Collection : Culture études

ISBN électronique : 9782111398832

\section{Sbooks}

http://books.openedition.org

\section{Édition imprimée}

Date de publication : 1 juin 2008

Nombre de pages : 12

\section{Référence électronique}

GOTTESDIENER, Hana ; VILATTE, Jean-Christophe ; et VRIGNAUD, Pierre. Image de soi-image du visiteur et pratiques des musées d'art. Nouvelle édition [en ligne]. Paris : Département des études, de la prospective et des statistiques, 2008 (généré le 25 avril 2021). Disponible sur Internet : <http:// books.openedition.org/deps/288>. ISBN : 9782111398832. 

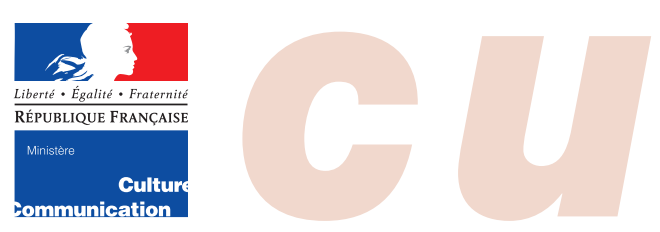

Secrétariat général

Délégation

au développement

et aux affaires

internationales

Département

des études,

de la prospective

et des statistiques

\section{Image de soi-image du visiteur et pratiques des musées d'art}

\author{
Hana Gottesdiener, \\ Jean-Christophe Vilatte, Pierre Vrignaud ${ }^{*}$
}

\section{Avant-propos}

Au moment où les établissements culturels comme tous les responsables de politiques de publics s'interrogent sur les conditions de relance des objectifs de démocratisation culturelle, il est fécond d'analyser les frontières qui dépassent, sans les contester, les puissants facteurs sociodémographiques des pratiques culturelles. La recherche présentée ici trouve sa place dans cette démarche. Son apport tient à la nouveauté de son analyse sur «l'image du visiteur», mais surtout à la comparaison qu'elle établit avec «l'image de soi » qui permet de rendre compte des pratiques muséales, notamment en ce qui concerne les musées d'art. La proximité ou la distance entre ces deux images sont en effet de nature non seulement à permettre de dégager des éléments prédictifs de la fréquentation, mais également à fournir un cadre d'interprétation plus général des comportements en matière de pratiques et/ou consommations culturelles légitimes. On constate une nouvelle fois que la médiation occupe une place centrale comme facteur de démocratisation culturelle, mais sur des perspectives renouvelées.

\section{Philippe Chantepie}

\section{Self-image/image of the gallery-goer, and art gallery practice}

Après avoir montré l'importance des facteurs sociodémographiques dans la fréquentation des institutions culturelles, les études menées sur les publics ont révélé celle des facteurs environnementaux, liés notamment aux paramètres de l'offre et aux modalités de fréquentation. Ce qui fait l'identité d'un individu et le poids de cette identité dans la définition des conduites sociales et dans leur régulation joue également un rôle central dans la fréquentation des musées. Si les liens entre cette fréquentation et les représentations ou l'image que les visiteurs se font des musées, de leurs collections, des outils de médiation ont fait l'objet de nombreuses études ${ }^{1}$, en revanche la question de l'image ou de la représentation de ce qu'est un visiteur de musée ne semble pas avoir été abordée ${ }^{2}$, peut-être parce que ces deux thématiques - image de soi et fréquentation sont traditionnellement traitées séparément, la première par la psychologie, la seconde, le plus souvent, par la sociologie descriptive ${ }^{3}$. Or, c'est une interrogation à envisager si l'on veut comprendre les ressorts des pratiques culturelles en général et de la visite des musées d'art en particulier.

\footnotetext{
* Hana GotTeSDiEnER est professeure émérite de psychologie à l'université Paris X-Nanterre, chercheuse au Laboratoire culture et communication de l'université d'Avignon et des Pays du Vaucluse, directrice et corédactrice en chef de la revue Culture \& Musées. Jean-Christophe VILATTE est maître de conférences en sciences de l'éducation à l'université Nancy 2 et chercheur au Laboratoire culture et communication de l'université d'Avignon et des Pays du Vaucluse. Pierre VRIGNAUd est professeur de psychologie à l'université de Paris X-Nanterre et chercheur au laboratoire Processus cognitifs et conduites interactives de la même université. Cette étude a été suivie par Sylvie Octobre au DePs.

1. David Alibert, Régis Bigot et Georges Hatchuel, Fréquentation et image des musées en début 2005, Paris, CREDOC, coll. «Rapports » $\left(\mathrm{n}^{\circ} \mathrm{R} 240\right)$, juin 2005 (document réalisé à la demande de la Direction des musées de France).

2. À partir de la consultation des principales bases de données en sciences humaines et sociales, il n'a pas été possible d'identifier des études sur les représentations du visiteur de musée, les mots clés «représentation» ou «image » renvoyant à des travaux sur les représentations du lieu muséal et de ses contenus.

3. L'interrogation des principales bases de données en sciences humaines et sociales en croisant ces deux thématiques n'a pas permis de mettre en évidence l'existence de travaux traitant de cette relation.
} 


\section{APPROChe NOUVELle : REPRÉSENTATIONS DU VISITEUR}

Tous les travaux de sociodémographie des publics ou de sociologie compréhensive qui se sont succédé ont mis en évidence le «plafond de verre ${ }^{4}$ » que constitue le « capital culturel » incorporé à l'individu qui accède aux équipements culturels ou à la culture en général : poids du diplôme, poids de la catégorie socioprofessionnelle, mais aussi poids des transmissions, mesurées souvent par l'origine sociale et le niveau de diplôme des parents; mise en évidence de facteurs « cachés » dans l'accession au savoir, les mêmes facteurs pesant sur l'accession aux cursus scolaires les plus prestigieux culturellement. Ces diagnostics étant avérés, la question des marges de manœuvre de l'action culturelle et éducative est alors posée.

Cette question se pose avec acuité dans le cas des jeunes publics, sur lesquels pèse le double pari de la socialisation culturelle : le pari des transmissions et de leur efficacité, qu'elles soient familiales, juvéniles, etc., et le pari de la médiation culturelle, qu'il s'agisse de l'école, des équipements culturels ou des lieux de diffusion et d'enseignement des arts. Elle se pose également, et ce avec particulièrement d'acuité, dans les équipements culturels. L'une de leurs missions fondamentales est en effet d'apporter leur concours à la transmission et l'éducation artistique et culturelle, et les efforts qui ont été consentis en la matière ont principalement porté sur l'éducation des jeunes publics, avec des résultats notoires en matière de connaissance des équipements culturels ${ }^{5}$.

Pour obtenir des réponses qui permettent de sortir de l'impasse, il faut donc changer de cadre d'analyse et porter attention non plus seulement aux phénomènes sociaux mais également aux phénomènes psychosociaux. Dans ce cadre, le concept de soi permet de donner une place à l'expérience sub- jective de l'individu, à la façon dont il se perçoit luimême, à ses attitudes, croyances et sentiments, à la façon dont il vit, structure et élabore son expérience interne et sa conduite en général ${ }^{6}$. Ainsi, le soi renvoie à des informations accumulées au cours de son existence, lesquelles renseignent sur ses capacités, ses réalisations, son potentiel et les aptitudes appelées à se manifester, ce qui lui permet de prendre des décisions, de choisir le comportement qu'il doit adopter face à une situation.

\section{Image de soi}

Les résultats de l'étude sur les freins et les motivations à la visite des musées d' $\operatorname{art}^{7}$ ont permis de mettre en évidence que la visite d'un musée d'art représente des risques pour un individu, selon que l'art fait partie ou non de son domaine culturel : le risque de la confrontation avec soi ou celui du passage dans un monde qui n'est pas le sien, le risque de la confrontation à l'œuvre qui conduit le visiteur à s'analyser comme sujet susceptible d'affirmer ses goûts, d'éprouver des émotions et d'exercer son sens critique, expérience que certains n'arrivent pas à éprouver et qui les met en situation d'échec.

On peut énoncer ainsi le modèle théorique sousjacent : lorsqu'un individu doit faire le choix d'une situation sociale ou, du moins, lorsqu'il doit exprimer une préférence vis-à-vis de cette situation, son choix résulte d'une stratégie d'appariement entre deux images : d'une part, les images de soi, sélectionnées par lui dans le répertoire d'images possibles de lui-même; d'autre part, l'image prototypique ou représentative des personnes dans la situation considérée ${ }^{8}$. Ce modèle, qui peut devenir prédictif des choix et préférences individuels, se résume de la façon suivante : plus la distance entre les deux représentations est faible, plus l'adoption du comportement est probable ${ }^{9}$.

4. Le concept de «plafond de verre» fut forgé à l’origine par les sociologues américains pour désigner les difficultés des femmes dans leur carrière professionnelle.

5. Sylvie Octobre, les Loisirs culturels des 6-14 ans, Paris, DePs, Ministère de la Culture et de la Communication/La Documentation française, 2004.

6. Winfrid HubER, Introduction à la psychologie de la personnalité, Bruxelles, Dessart \& Mardaga, 1977.

7. Hana GotTeSdiener, Freins et motivations à la visite des musées d'art, Paris, Deps, Ministère de la Culture, 1992.

8. Michel HuteAu, Françoise VouILLOT, « Représentations et préférences professionnelles », Bulletin de psychologie, XLII (388), 1988, p. 144153.

9. Pour illustrer cette démarche, on peut prendre l'exemple de la méthode utilisée dans l'étude de M. Huteau et F. Vouillot (voir note 8) sur les représentations et préférences professionnelles de lycéens. Ces derniers sont invités, d'une part, à se décrire et, d'autre part, à décrire six métiers à l'aide de 58 adjectifs, tirés d'un questionnaire de personnalité l'Adjective Check List (ACL) de Gough (1982), adjectifs dont on considère qu'ils décrivent de manière pertinente des professions. Ces lycéens doivent par ailleurs exprimer leur degré d'attrait par rapport aux six métiers sur une échelle en quatre points. Pour chaque sujet, une distance entre l'image de soi et la représentation du métier est calculée. Chaque distance est ensuite mise en relation avec le degré d'attrait pour le métier considéré. Cette étude montre que plus la distance entre l'image de soi et la représentation du métier est faible (les mêmes adjectifs sont utilisés pour décrire les deux) et plus l'attrait pour le métier est fort. D'autres études portant sur d'autres objets sociaux et obtenant les mêmes résultats permettent de conclure que la stratégie d'appariement est assez générale et que l'on peut prédire des préférences pour des situations et rôles sociaux assez divers. 


\section{Image du visiteur de musée...}

Le champ muséal est un objet d'étude privilégié pour ce nouveau type d'analyse, pour une double raison : la prégnance des images ou représentations sociales que cette pratique dite «légitime » suscite, et celle des images ou des représentations produites sur ceux qui s'y adonnent. Dans l'ensemble, en effet, qu'ils fréquentent ou pas les musées, la représentation que les individus en ont est positive, comme le montrent les statistiques : les musées sont perçus par $89 \%$ des Français comme des lieux où l'on apprend toujours quelque chose, $67 \%$ d'entre eux considèrent qu'aller au musée est un « vrai plaisir » et $59 \%$ qu'il s'agit de lieux «bien aménagés pour le confort des visiteurs », alors que seulement $33 \%$ d'entre eux y sont allés au cours des douze mois précédents l'enquête ${ }^{10}$. L'image positive des musées ne semble donc pas être suffisante pour rendre compte de la fréquentation puisque certains ne visitent pas de musée alors qu'ils en ont une image positive. Pour justifier le fait de ne pas visiter de musée, $43 \%$ des personnes interrogées déclarent que cela ne les "intéresse pas vraiment», manque d'intérêt qui concerne toutes les catégories sociales, notamment les individus plutôt traditionalistes en matière de mœurs, « économes » vis-à-vis de certaines pratiques, souvent inquiets et disposant d'un réseau de relations assez faible.

\section{... et distance ou proximité entre les deux images}

Si l'image qu'un individu a des musées ne permet pas de prédire sa fréquentation, l'image qu'il a de lui-même confrontée à celle qu'il a du visiteur de musée peut être susceptible de l'expliquer. On constate en effet que plus les deux images sont proches, plus il est possible de prédire qu'il y aura fréquentation des musées : si l'individu s'attribue les caractéristiques du visiteur, il visite les musées en pensant que ce sont des lieux qui lui correspondent. À l'inverse, plus les deux images sont distinctes, plus improbable pourrait être la visite.

Les résultats obtenus dans le champ des musées sont de nature à fournir un cadre permettant une interprétation plus large des comportements en matière d'autres pratiques et/ou consommations culturelles légitimes.

\section{DisTANCE ET PROXIMITÉ...} PRATIQUE DES MUSÉES,

\section{PERCEPTION DE SOI ET DES VISITEURS}

L'enquête ayant permis de mettre en regard la distance entre les deux profils d'image, de soi et du visiteur de musée des beaux-arts, et la pratique des musées, il est possible de faire un lien entre les représentations et les comportements des personnes enquêtées. La pratique a été évaluée à partir de questions relatives à la fréquence de visite des lieux d'art, à l'importance accordée à la fréquentation des musées, à la compétence à juger de la qualité d'une exposition ou à donner des conseils aux autres quant à la visite d'un lieu d'art, et enfin à partir de la consultation de sites internet dédiés aux musées (voir encadré méthodologique p. 10).

\section{Liens avec la probabilité de visite...}

Il s'agit d'analyser la relation observée entre, d'une part, la distance moyenne entre image de soi et image du visiteur se dégageant pour 24 adjectifs (12 dits « pertinents » qualifiant le visiteur de musée d'art et 12 dits « distracteurs ${ }^{11} »$, voir encadré ciaprès) et, d'autre part, la fréquentation des musées des beaux-arts et des musées d'art moderne et contemporain au cours de l'année précédant l'enquête.

\begin{tabular}{|c|c|c|c|}
\hline \multicolumn{4}{|c|}{ Les adjectifs } \\
\hline \multicolumn{2}{|c|}{$\begin{array}{c}\text { dits « pertinents " (12) } \\
\text { décrivant } \\
\text { le visiteur de musée }\end{array}$} & \multicolumn{2}{|c|}{$\begin{array}{c}\text { dits « distracteurs » (12) } \\
\text { ne décrivant pas } \\
\text { particulièrement } \\
\text { le visiteur de musée }\end{array}$} \\
\hline Artiste & Passionné & Actif & Gai \\
\hline Attentif & Rêveur & Direct & Généreux \\
\hline Calme & Sensible & Dynamique & Organisé \\
\hline Cultivé & Tolérant & Efficace & Persévérant \\
\hline Curieux & Ouvert & Exigeant & Perspicace \\
\hline Imaginatif & Patient & Précis & Rationnel \\
\hline
\end{tabular}

Si l'on examine les distances moyennes « image de soi-image du visiteur des musées des beauxarts » pour l'ensemble des 12 adjectifs pertinents en fonction de la fréquentation des musées, on constate que pour les individus ayant visité au minimum trois musées des beaux-arts et trois musées d'art moderne et contemporain cette distance est positive alors qu'elle est négative pour ceux qui n'en ont pas visités (voir tableau 1).

10. D. Alibert, R. Bigot et G. Hatchuel, Fréquentation et image des musées en début 2005, op. cit.

11. En ce qui concerne les adjectifs dits « distracteurs », s'il n'est guère possible d'interpréter les différences observées entre image de soi et image du visiteur du point de vue des différentes caractéristiques des personnes enquêtées dont on se propose d'étudier les effets, on peut éventuellement observer des distances positives entre les deux images, les individus étant susceptibles de s'attribuer ce qu'ils considèrent être des qualités de manière générale. Rappelons que l'emploi des adjectifs « distracteurs » permet de contrôler que les réponses à l'ensemble des tâches proposées sont valides (voir encadré méthodologique p. 10). 
Tableau 1 - Relations entre la moyenne des distances image de soi-image du visiteur de musée des beaux-arts et la fréquentation des musées d'art

\begin{tabular}{|c|c|c|c|}
\hline \multirow[b]{2}{*}{$\begin{array}{l}\text { Fréquentation des musées d'art } \\
\text { au cours de l'année précédant l'enquête }\end{array}$} & & \multicolumn{2}{|c|}{ e soi-visiteur pour les 12 a } \\
\hline & & "pertinents " & « distracteurs " \\
\hline “Ont visité..." & $\mathbf{N}$ & Moyenne des distances & Moyenne des distances \\
\hline \multicolumn{4}{|l|}{ un musée des beaux-arts } \\
\hline Non & 131 & $-5,75$ & 6,97 \\
\hline Une ou deux fois & 104 & $-3,34$ & 7,73 \\
\hline 3 fois et plus & 77 & 3,01 & 8,05 \\
\hline \multicolumn{4}{|l|}{ un musée d'art moderne et contemporain } \\
\hline Non & 146 & $-6,53$ & 7,48 \\
\hline Une ou deux fois & 101 & $-1,74$ & 6,95 \\
\hline 3 fois et plus & 60 & 4,29 & 8,35 \\
\hline
\end{tabular}

Source : DEPS, Ministère de la Culture et de la Communication
Ce tableau permet de constater que dans le cas où la fréquentation des musées d'art est plutôt assidue, les individus se reconnaissent dans les traits qu'ils attribuent au visiteur de musée, et que dans le cas où la pratique de visite est nulle ou faible, c'est le contraire. Quelle conclusion en tirer? L'individu fréquente-t-il les musées parce qu'il se définit comme un visiteur de musée, du moins une fois la pratique installée ? L'image de soi obtenue à partir des adjectifs proposés est dans ce cas conforme à l'image prototypique du visiteur de musée et l'on peut penser que plus l'individu va au musée et plus il se perçoit comme ayant, à un haut niveau, les qualités attribuées à ce que l'on considère comme un visiteur moyen. Si l'image de soi est trop éloignée de celle du visiteur, la décision de visiter un musée est sans doute plus difficile à provoquer ${ }^{12}$.

À fréquentation équivalente, des distinctions apparaissent selon le type de musée, les visiteurs des musées d'art contemporain se percevant en effet comme détenteurs des qualités affectées aux visiteurs de musée à un degré plus important que ceux des musées des beaux-arts. Cette observation est cohérente avec les résultats de l'étude sur les jeunes adultes et l'art contemporain ${ }^{13}$ : il y apparaissait que les jeunes adultes fréquentant les musées d'art moderne et contemporain ont des pratiques de visite et des goûts plus diversifiés et plus intenses que ceux qui visitent les musées des beaux-arts.
En ce qui concerne les visiteurs des musées des beaux-arts occasionnels - entre 1 et 2 visites -, ils se comportent comme ceux qui ne fréquentent pas les musées, ce résultat étant en cohérence avec ce que l'on sait par ailleurs des pratiques culturelles. Cette observation confirme l'existence de « seuils » psychologiques, nécessitant que la pratique soit répétée. Pour les musées des beaux-arts, on constate cet effet de seuil pour une fréquentation non exceptionnelle -3 visites annuelles ou plus - dans le cas de 7 adjectifs pertinents sur 12 : artiste, attentif, cultivé, curieux, imaginatif, passionné, rêveur. Ainsi, dans le cas d'une fréquentation régulière, l'individu se pense doté des mêmes qualités que celles des visiteurs de musée et fait dès lors partie du même univers qu'eux.

Le calme et la patience sont des qualités intrinsèquement attachées à l'image du visiteur de musée, qualités qui, d'une certaine façon, ne sont pas conçues comme pouvant s'acquérir au même degré, alors que les qualités proprement individuelles, non affectées par la fréquentation des musées, sont la tolérance, la sensibilité et l'ouverture. Il ressort en effet de l'enquête que l'individu, quelle que soit sa fréquentation, a tendance à considérer le visiteur de musée des beaux-arts plus calme et plus patient que lui-même, et qu'à l'inverse, il se perçoit plus tolérant et plus sensible, mais également plus ouvert, que celui-ci.

12. Quant aux adjectifs distracteurs, on constate que les valeurs de la distance moyenne image de soi-image du visiteur de musée des beauxarts sont proches et positives, quelle que soit la fréquentation des musées des beaux-arts ou des musées d'art moderne ou contemporain au cours de l'année précédant l'enquête. Conformément à l'hypothèse qui avait été formulée en ce qui concerne ces adjectifs, il n'existe pas de relation entre les deux profils d'images et la fréquentation. Par ailleurs, on peut observer que les personnes enquêtées ont une perception d'euxmêmes qui est positive. Ce résultat est tout à fait cohérent : il n'y a en effet aucune raison que, pour des adjectifs qui ne décrivent pas spécialement le visiteur de musée mais qui renvoient à des traits de personnalité positifs (exemple : être gai, généreux, actif...), les personnes enquêtées se sous-évaluent par rapport au visiteur de musée.

13. Hana GotTESDIENER, Jean-Christophe VILATTE, l'Accès des jeunes adultes à l'art contemporain. Approches sociologique et psychologique du goût des étudiants pour l'art et de leur fréquentation des musées, Paris, Ministère de la Culture et de la Communication, coll. «Les travaux du DEPS », 2006. 
On peut interpréter la valorisation de ces adjectifs comme un phénomène de «désirabilité sociale $^{14} »$ : il est en effet socialement plus accepté d'être tolérant et ouvert plutôt qu'intolérant et fermé. En revanche, la valorisation de l'adjectif « sensible » vis-à-vis de l'image de soi est plus délicate à interpréter : les personnes interrogées dans le cadre de l'enquête ont-elles attribué le même sens à cet adjectif lorsqu'elles ont eu à juger de la pertinence de cette qualité vis-à-vis d'elles-mêmes et vis-à-vis du visiteur de musée ? La notion de sensibilité recouvre en effet différentes formes dont l'une se rattache plus particulièrement à l'art et si certains pensent à la sensibilité artistique dans le cas des visiteurs, ils pensent sans doute à la sensibilité en général dans leur propre cas.

Pour interpréter l'ensemble des résultats de l'enquête, deux types de relations doivent être analysés : entre image du visiteur et fréquentation, et entre image de soi et fréquentation. Si l'on constate qu'il y a bien un lien dans le premier cas - l'image du visiteur diffère selon la fréquentation ${ }^{15}-$, par contre l'image de soi n'est pas en relation avec celle-ci ${ }^{16}$. L'image du visiteur paraît donc être modifiable plus facilement que l'image de soi, laquelle est toujours plus générale ${ }^{17}$.

Ainsi peut-on comprendre les variations introduites par le fait de fréquenter ou non les musées : le non-visiteur idéalise le visiteur de musée en lui attribuant de nombreuses qualités qu'il considère comme nécessaires à la visite, mais ne s'en attribue à lui-même aucune; en revanche, le visiteur de musée se perçoit comme ayant à un plus haut degré les qualités attribuées à un visiteur qu'il n'idéalise pas.

Ces résultats montrent bien que le frein à l'élargissement des publics est d'ordre psychosociologique et qu'il tient aussi aux représentations que les publics peu familiers des musées ont d'eux-mêmes avant de tenir aux représentations qu'ils ont des musées.

\section{... avec la probabilité de consultation des sites de musée}

La création de sites virtuels par les musées et leur multiplication se sont fondées, de façon implicite ou explicite, sur l'hypothèse que ce ne sont pas les mêmes facteurs qui président à la visite d'un musée et à la consultation d'un site sur l'internet. Selon des études portant sur la sociographie des usagers, les publics virtuels sont en général plus jeunes et ont un niveau de diplôme plus élevé que ceux qui fréquentent les musées, les deux phénomènes étant liés.

La recherche présentée ici permet-elle de dire que la fréquentation des sites web de musée met en jeu des images de soi et des visiteurs de musée différentes de ce qui se passe dans le cas de la visite réelle ? Comme le montre le tableau 2, visite virtuelle et visite réelle sont liées. Près des trois quarts

\section{Tableau 2 - Visite de musée des beaux-arts et consultation de sites de musée des beaux-arts}

\begin{tabular}{|c|c|c|c|c|c|c|c|c|c|}
\hline \multirow{3}{*}{$\begin{array}{l}\text { Avez-vous visité } \\
\text { un musée des beaux-arts? }\end{array}$} & \multicolumn{9}{|c|}{ Nombre de visites de sites web de musée des beaux-arts } \\
\hline & \multicolumn{2}{|c|}{ Non } & \multicolumn{2}{|c|}{1 à 3 fois } & \multicolumn{2}{|c|}{4 à 9 fois } & \multicolumn{2}{|c|}{10 fois et plus } & \multirow{2}{*}{$\begin{array}{c}\text { Total } \\
\mathrm{N}\end{array}$} \\
\hline & $\mathrm{N}$ & $\%$ & $\mathrm{~N}$ & $\%$ & N & $\%$ & N & $\%$ & \\
\hline Non & 112 & 72,2 & 38 & 24,50 & 4 & 2,5 & 1 & 0,6 & 155 \\
\hline 1 ou 2 fois & 65 & 52,8 & 34 & 27,60 & 19 & 15,4 & 5 & 4,0 & 123 \\
\hline 3 fois et plus & 14 & 19,4 & 6 & 8,33 & 10 & 13,8 & 42 & 58,3 & 72 \\
\hline Total & 191 & 54,7 & 78 & 22,20 & 33 & 9,4 & 48 & 13,7 & 350 \\
\hline
\end{tabular}

14. L'échelle de désirabilité sociale (J. TouRnors, F. MEsnil et J.-L. Kop, «Autoduperie et hétéroduperie : un instrument de mesure de la désirabilité sociale», European Review of Applied Psychology, 2000, 50, p. 219-233) permet d'évaluer la susceptibilité des personnes à être influencées par le niveau de désirabilité sociale de leurs réponses. Alors que certaines personnes répondent vraiment aux questions en fonction de leur réalité, d'autres ont tendance à modifier leurs réponses afin qu'elles soient plus acceptables socialement.

15. Nous prendrons deux exemples : pour le qualificatif «artiste», la moyenne est de 3,5 pour ceux qui ne vont pas dans un musée des beauxarts et de 2,8 pour ceux qui y vont au moins trois fois par an; pour «attentif» les valeurs sont respectivement de 4,4 et 3,5.

16. Si nous reprenons les deux mêmes exemples, nous constatons que la différence est faible. Pour «artiste», on a 2,4 et 2,9 et pour « attentif », on a 3,8 et 3,7 .

17. Il est à rappeler qu'avant de remplir la première partie du questionnaire qui portait sur la description de soi, les enquêtés étaient simplement informés que l'étude portait sur leur intérêt pour certaines activités culturelles. 
de ceux qui ne sont jamais allés au musée des beaux-arts au cours de l'année n'ont jamais non plus visité un site de musée des beaux-arts au cours de la même période. Inversement, près de la moitié de ceux qui sont allés 1 à 2 fois dans un musée des beaux-arts au cours de l'année ont consulté un site de musée des beaux-arts : $27 \%$ une à trois fois, $15 \%$ entre 4 et 9 fois et $4 \%$ dix fois et plus.

Avec un peu plus de la moitié de l'échantillon $(53,6 \%)$, la pratique «consultation de sites » de musée des beaux-arts se situe au niveau de la fréquentation du musée lui-même (32\% ont déclaré avoir visité un musée des beaux-arts entre une et deux fois et $23 \%$ trois fois et plus). Cette pratique semble relativement bien installée chez certaines personnes, comme le montre le nombre de sites de musée des beaux-arts visités : $22 \%$ déclarent avoir consulté au moins trois fois un site, $9 \%$ entre quatre et neuf fois et $14 \%$ dix fois et au-delà. Mais l'enquête n'a pas permis de connaître les motifs de consultation : consulter un programme ? Vérifier un horaire ? Visiter la collection virtuelle du musée ?...

S'il ressort que ces pratiques sont liées, sontelles affectées par les mêmes effets de distance ou de proximité entre image de soi et image du visiteur de musée ? Comme le montre le tableau 3, pour ceux qui ne fréquentent pas les sites web, la distance image de soi-image du visiteur de musée des beaux-arts est négative $(-6,3)$ alors qu'elle est positive $(0,43)$ pour ceux qui les fréquentent. Par ailleurs, la distance est plus faible chez ceux qui les fréquentent plus souvent, et l'on note que c'est audelà de 10 consultations de sites qu'elle devient positive. Les résultats sont parfaitement ordonnés, les distances diminuent régulièrement entre ceux qui ne visitent pas et ceux qui ont fréquenté entre 4 et 9 musées.

Les mêmes mécanismes psychosociologiques président donc aux visites des musées eux-mêmes et à la fréquentation des sites de musée. Ceux qui visitent souvent un site se perçoivent comme ayant, à un niveau plus élevé, les qualités attribuées au visiteur de musée alors que c'est l'inverse dans le cas où il n'y a pas consultation de sites ou seulement de manière occasionnelle. L'arrivée des technologies n'a donc pas modifié fondamentalement le rapport des publics aux musées mais assure une fonction d'amplification.

\section{Importance accordée aux musées et compétence auto-attribuée}

Si l'on examine l'importance que les personnes enquêtées accordent aux visites de musée et la compétence qu'elles s'attribuent pour juger de la qualité d'une exposition ou pour exprimer un avis en matière d'expositions auxquelles aller, voit-on apparaître les mêmes effets de seuil que ceux apparus en matière de fréquentation de musée et de consultation de sites?

Il semble que les choses sont moins nettes (voir tableau 4). Si l'on considère par exemple les liens entre importance accordée aux visites et image de soi-image de visiteur de musée, on observe que la distance moyenne entre les deux profils d'images est bien négative quand elles considèrent qu'il n'est pas du tout important de fréquenter les musées et que cette distance diminue au fur et à mesure qu'elles accordent de l'importance à la visite, mais cette distance ne devient positive que lorsque cette visite est jugée très importante et, même dans ce cas, elle reste relativement faible $(0,92)$.

Compte tenu de ces résultats, il était prévisible que la distance serait positive entre image de soi et image du visiteur pour les individus déclarant que « visiter des musées est important » pour eux : il est en effet socialement délicat d'avouer le contraire, si bien que quelqu'un peut répondre «c'est important », qu'il les fréquente ou pas. Or, seuls ceux qui répondent «il est très important de visiter les musées » peuvent être considérés comme étant des visiteurs assidus, du moins dans l'échantillon de l'enquête présentée ici, d'où la distance positive observée pour cette catégorie de sujets.

Quel que soit le critère retenu (importance accordée à la fréquentation des musées, compétence à

\section{Tableau 3 - Relations entre la distance image de soi-image du visiteur et visite de sites de musée des beaux-arts}

\section{Avez-vous visité un site de musée des beaux-arts au cours des 12 mois précédant l'enquête?}

Non

De 1 à 3 fois

De 4 à 9 fois

10 fois et plus

Distance soi-visiteur pour les 12 adjectifs dits «pertinents » «distracteurs"

Moyenne des distances
$-5,72$
$-2,86$
$-0,31$
4,40

Moyenne des distances N 72 29 45 
Tableau 4 - Relations entre moyenne des distances image de soi-image du visiteur de musée des beaux-arts et importance de visiter des musées

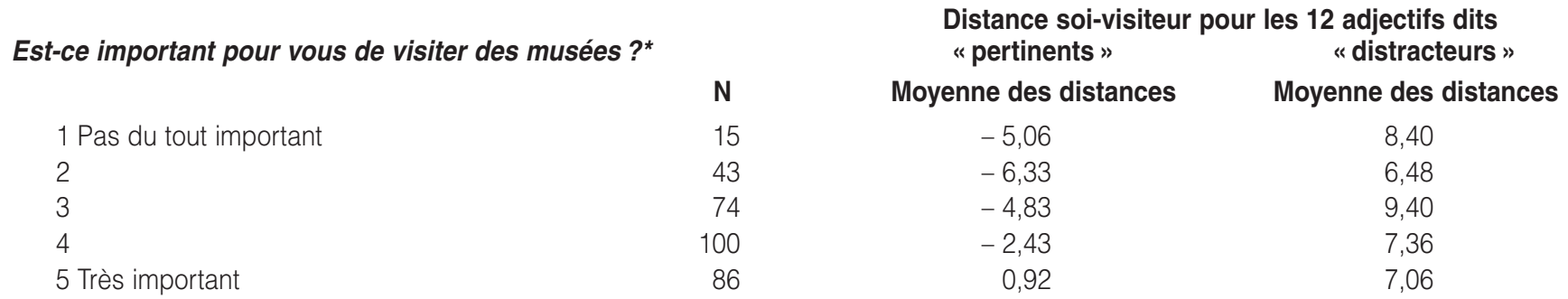

${ }^{*}$ Les personnes enquêtées devaient cocher la case correspondant à leur jugement sur une échelle en 5 points, de $1=$ pas du tout important à $5=$ très important.

Source : DEPS, Ministère de la Culture et de la Communication

juger de la qualité d'une exposition ou à donner des conseils quant à la visite d'un lieu d'art), il semble bien que la compétence que l'individu s'attribue soit en lien avec la distance image de soi-image du visiteur, même si ce lien est moins fort que dans le cas de la fréquentation des musées ou de la consultation des sites internet.

\section{DES VARIATIONS LIÉES}

\section{AUX PROFILS SOCIODÉMOGRAPHIQUES}

Si l'image du visiteur est fortement liée à la fréquentation des musées, on devrait donc retrouver l'influence de certaines variables sociodémographiques - formation, genre ou niveau d'études de la mère par exemple -, sur la distance image de soiimage du visiteur, puisque ces variables opèrent de façon puissante dans le champ des pratiques muséales.

\section{L'effet des filières de formation}

Si l'on considère, filière par filière, la relation entre image de soi et image du visiteur de musée des beaux-arts, on observe que les distances entre les profils des deux images varient de manière non arbitraire en fonction de la nature de la filière (voir tableau 5).

Les résultats de l'enquête montrent que pour les individus qui ont suivi une formation en histoire de l'art (École du Louvre, Louvre master), la distance moyenne image de soi-image du visiteur est positive alors que pour les autres, elle est négative, et dans ce cas, elle est d'autant plus grande que ces derniers ont suivi une filière scientifique et technique (génie électrique et licence professionnelle ${ }^{18}$, voir tableau 5). Ceux qui ont suivi une formation dans le domaine de l'art ont donc tendance à s'attribuer les adjectifs qui qualifient le visiteur de musée plus encore qu'ils ne les attribuent au visiteur de musée des beaux-arts : ainsi, les étudiants de l'École du Louvre qui fréquentent les musées en raison de leur formation sont les seuls à se percevoir plus «artistes », «attentifs », «calmes » et «cultivés» que les visiteurs de musée des beauxarts, alors que tous les autres étudiants considèrent que ces qualités caractérisent mieux ces visiteurs qu'eux-mêmes.

Pour l'ensemble des autres filières, il semble que l'image de soi se rapproche de l'image du visiteur

\section{Tableau 5 - Relations entre la moyenne des distances image de soi-image du visiteur de musée des beaux-arts et la filière suivie}

\begin{tabular}{|c|c|c|c|}
\hline \multirow[b]{3}{*}{ Psychologie $1^{\text {re }}$ année } & \multirow{3}{*}{$\begin{array}{l}\mathbf{N} \\
39\end{array}$} & \multicolumn{2}{|c|}{$\begin{array}{c}\text { Distance soi-visiteur } \\
\text { pour les } 12 \text { adjectifs dits } \\
\text { pertinents » « distracteurs » }\end{array}$} \\
\hline & & \multicolumn{2}{|c|}{ Moyenne des distances } \\
\hline & & $-5,07$ & 4,85 \\
\hline Psychologie $2^{\mathrm{e}}$ année & 41 & $-7,21$ & 4,41 \\
\hline Sciences éducation & & & \\
\hline licence & 26 & $-3,26$ & 9,76 \\
\hline $\begin{array}{l}\text { Sciences éducation } \\
\text { master }\end{array}$ & 18 & $-2,61$ & 5,81 \\
\hline Génie électrique & 54 & $-7,24$ & 7,43 \\
\hline $\begin{array}{c}\text { Centre d'éducation } \\
\text { permanente }\end{array}$ & 36 & 0,13 & 8,47 \\
\hline Individuels (Parisiens) & 30 & $-2,80$ & 6,00 \\
\hline $\begin{array}{l}\text { Sciences éducation } \\
\text { licence pro }\end{array}$ & 9 & $-10,44$ & 13,57 \\
\hline $\begin{array}{l}\text { Sciences éducation } \\
\text { master pro }\end{array}$ & 28 & $-3,92$ & 10,84 \\
\hline École du Louvre & 50 & 5,64 & 10,32 \\
\hline Louvre master & 15 & 6,73 & 5,50 \\
\hline Individuels (Messins) & 7 & $-10,14$ & 9,57 \\
\hline
\end{tabular}

Source : DEPS, Ministère de la Culture et de la Communication

18. Les étudiants de la licence professionnelle sciences de l'éducation sont des professionnels en reconversion, issus majoritairement de branches professionnelles du technique ou du commercial. 
lorsque le niveau de formation s'élève ou lorsque l'enquêté est âgé, même si la distance reste négative. S'il y a rapprochement, c'est qu'il y a à la fois modification de l'image du visiteur et modification de l'image de soi, dont le qualificatif «artiste» fournit un exemple : quand la formation s'élève, la personne enquêtée perçoit le visiteur comme moins artiste et se perçoit comme un peu plus artiste, ce qui tend à montrer qu'il y a à la fois moins de stéréotypie $^{19}$ et plus d'affirmation de soi ${ }^{20}$.

Il semble que l'effet «formation » joue de la même manière sur l'image du visiteur que l'effet « fréquentation ${ }^{21} »$ : ceux qui ont une formation artistique ont globalement une image du visiteur moins positive que les autres. Il semblerait en revanche que ce ne soit pas le cas pour l'image de soi puisque l'on observe un effet de la formation qui n'apparaît pas dans le cas de la fréquentation. L'image de soi semble donc plus sensible à la filière de formation, ce qui renforce l'idée énoncée précédemment: il est difficile de modifier l'image globale de soi par la pratique de visite.

\section{L'effet du genre}

Si l'on considère les enquêtes conduites dans les musée ${ }^{22}$, les musées d'art sont fréquentés davantage par les femmes que par les hommes ${ }^{23}$, alors que les enquêtes sur les pratiques culturelles ne montrent pas de différence en ce qui concerne la visite des musées des beaux-arts ${ }^{24}$. On y constate que si les hommes sont moins nombreux que les femmes à déclarer visiter une exposition de peinture, ils sont plus nombreux à dire être allés dans une exposition de photographies et dans une galerie d'art.

En ce qui concerne l'enquête présentée ici, les personnes interrogées dans leur ensemble fréquentent peu les musées. Comme cela a été constaté plus haut, on constate une distance négative entre image de soi et image du visiteur, quel que soit le genre, bien que ce facteur joue un rôle puisque cette distance est plus importante pour les hommes que pour les femmes (respectivement $-4,58$ et $-1,99$ ). Cette observation montre que les femmes ont tendance à s'attribuer les caractéristiques du visiteur de musée davantage que les hommes et corrobore à ce titre la partition sexuée du monde des musées et en particulier des représentations des visiteurs des musées des beaux-arts.

\section{L'effet du niveau d'études de la mère}

Les enquêtes sur les publics des musées ont également mis en évidence l'effet du niveau d'études des parents, notamment de la mère, sur la probabilité pour l'enfant de fréquenter les musées. Se pose alors une question: y a-t-il pareillement un effet «niveau d'études de la mère » sur les représentations et sur le lien image de soi-image du visiteur

On constate que plus le niveau d'études de la mère est élevé, plus la distance entre les deux profils d'image est faible ou, pour le dire autrement, plus l'image de soi est décrite comme étant proche de celle du visiteur de musée des beaux-arts (voir tableau 6). Ce résultat confirme les travaux de sociologie descriptive qui mettent en évidence l'existence d'une relation entre le niveau d'instruction de la famille et les pratiques artistiques et culturelles. On observe ainsi que le niveau d'instruction des parents joue davantage que le niveau d'instruction de l'enquêté lui-même dans son orientation

\section{Tableau 6 - Relations entre la moyenne des distances image de soi-image du visiteur de musée des beaux-arts et niveau d'études de la mère}

\begin{tabular}{|c|c|c|c|}
\hline \multirow[b]{2}{*}{$\begin{array}{l}\text { Niveau } \\
\text { d'études de la mère }\end{array}$} & \multicolumn{3}{|c|}{$\begin{array}{c}\text { Distance soi-visiteur } \\
\text { pour les } 12 \text { adjectifs dits } \\
\text { «pertinents « «distracteurs » }\end{array}$} \\
\hline & $\mathbf{N}$ & \multicolumn{2}{|c|}{ Moyenne des distances } \\
\hline En dessous & & & \\
\hline du baccalauréat & 152 & $-4,61$ & 7,71 \\
\hline Bac ou équivalent & 74 & $-2,93$ & 7,42 \\
\hline Études supérieures & 120 & $-0,61$ & 7,64 \\
\hline
\end{tabular}

19. Image du visiteur «artiste » : quand on passe du niveau DEUG au niveau master, la moyenne est de 4,31 pour psychologie ou de 4,05 pour génie électrique et de 3,45 pour master sciences de l'éducation.

20. Image de soi : la moyenne pour la psychologie est de 2,36 ou de 2,60 pour génie électrique et de 3,05 pour le master sciences de l'éducation.

21. Notons que la formation à tendance à maximiser l'écart. Ainsi, pour le qualificatif «artiste», dans le cas de l'image du visiteur, l'écart entre les moyennes pour les étudiants de l'École du Louvre et du génie électrique est de 1,53 point (la moyenne pour le génie électrique est de 4,05 et de 2,52 pour l'École du Louvre) et seulement de 0,7 point dans le cas de la comparaison entre ceux qui visitent et ceux qui ne visitent pas.

22. Lucien Mironer, Cent musées à la rencontre du public, Cabestany, France Édition, 2001.

23. Dans les enquêtes conduites dans les musées, les questionnaires sont le plus souvent auto-administrés et la question de la disposition à répondre à ces questionnaires selon le genre peut être posée.

24. Olivier Donnat, les Pratiques culturelles des Français. Enquêtes 1997, Paris, Deps, Ministère de la Culture/La Documentation française, 1998. 
vers des lieux culturels et dans ses goûts en matière de peinture ${ }^{25}$.

Toutefois, l'influence du niveau d'études parental, et notamment maternel, semble avoir des effets limités dans le cas de la comparaison image de soiimage du visiteur. On aurait pu s'attendre en effet à trouver une distance moyenne positive entre image de soi et image du visiteur de musée des beaux-arts dans le cas des individus ayant une mère ayant fait des études supérieures car ils devraient se percevoir comme ayant à un plus haut degré les qualités du visiteur de ces musées. Le niveau d'études des parents semble donc moins discriminant que les études poursuivies ou que la fréquentation actuelle.

\section{LA CONSTRUCTION D'UNE IMAGE}

\section{DE VISITEUR MOINS IDÉALISÉE :}

\section{CONDITION DE LA DÉMOCRATISATION DES MUSÉES D'ART ?}

La comparaison entre image du visiteur et image de soi met en lumière l'existence d'une relation avec la pratique muséale. Les personnes de l'échantillon qui ont une forte pratique des musées s'approprient les adjectifs qui décrivent le visiteur plus qu'ils ne les attribuent au visiteur de musée des beaux-arts, alors que celles qui n'ont pas de pratique, ou dont la pratique est faible, ont tendance à se sous-estimer par rapport à l'image qu'elles se font du visiteur de musée.

D'autres facteurs, comme le niveau de diplôme, le type d'études, le niveau d'études des parents, jouent également un rôle. Plus le niveau est élevé et plus l'image de soi est proche de l'image du visiteur : les niveaux les plus élevés s'attribuent davantage les adjectifs décrivant le visiteur de musée qu'ils ne les attribuent au visiteur lui-même et se perçoivent plus visiteur que le visiteur type des musées, ceci étant particulièrement net pour les étudiants en art.

L'analyse des images négatives que les personnes enquêtées ont des musées a permis de tester une série d'incitations (gratuité des musées, prix d'entrée, mise à disposition d'informations sur le contenu des musées et expositions, horaires...) et de souligner la relative inertie des comportements face à ces incitations ${ }^{26}$. La prise en compte de l'image de soi et de sa distance à l'égard de l'image des visiteurs de musée fournit des éléments pour interpréter ce constat : plus la pratique muséale de l'individu est faible, voire inexistante, plus il se perçoit différent du visiteur de musée, considérant ne pas avoir les qualités de ce dernier. Ainsi, le nonvisiteur ou le visiteur occasionnel se considère moins curieux, moins passionné, moins cultivé, moins attentif... que le visiteur de musée. Autrement dit, les publics ont une perception a priori de leur inadéquation au champ muséal: dans ces conditions, franchir les portes d'un musée suppose une série d'efforts (cognitifs, conatifs, etc.) dont le coût perçu semble élevé.

La réponse que peut alors proposer l'institution muséale n'est pas de nier cet effort en proposant une approche qui se voudrait ludique ou agréable au risque de ne pas être crédible auprès de ce public, mais de l'accompagner, de l'aider à mieux l'accepter sans, pour autant, idéaliser le visiteur et ce qu'est la visite d'un musée.

Des études antérieures portant sur les freins et motivations à la visite des musées d'art ${ }^{27}$ et sur l'impact du dimanche gratuit au musée du Louvre ${ }^{28}$ ont montré la nécessité d'accompagner le visiteur dans sa démarche de franchissement. Les résultats de la recherche présentée ici rendent encore plus évidente la nécessité de s'interroger sur ce que pourrait être un accompagnement qui permettrait d'apprendre à visiter et de réduire la distance entre la perception de soi et la perception de l'image du visiteur. À cet égard, on peut penser à des médiations qui mettent des visiteurs occasionnels en situation d'exercer les compétences qu'ils attribuent au visiteur de musée, et surtout d'en faire l'analyse. On peut faire l'hypothèse que la prise de conscience par un individu qu'il est capable, dans le contexte du musée, de faire preuve de curiosité, d'attention, d'imagination, peut modifier, dans un premier temps, l'image qu'il a du visiteur et sa propre pratique, et peut-être, dans un second temps, et seulement après des expériences renouvelées, l'image de soi.

25. Yvonne BERnARD, Psycho-sociologie du goût en matière de peinture, Paris, CNRS, coll. « Monographie française de psychologie », $\mathrm{n}^{\circ} 24$, 1973.

26. D. Alibert, R. Bigot et G. Hatchuel, Fréquentation et image des musées en début 2005, op. cit.

27. Hana GotTesdiener, Freins et motivations à la visite des musées d'art, Paris, DePs, Ministère de la Culture, 1992.

28. Hana GotTesdiener, Nathalie GodrèCHE, «Perceptions et comportements de visite lors des dimanches gratuits au musée du Louvre », dans Claude Fourteau, la Gratuité au bois dormant... Cinq ans de gratuité du dimanche au Louvre 1996-2000, Paris, Musée du Louvre, 2002, p. 37-109. 


\section{MÉTHODOLOGIE}

\section{L’approche}

L'analyse de la relation entre image de soi et image du visiteur de musée d'une part et pratiques muséales de l'autre repose sur un outil de mesure permettant aux enquêtés soit de se décrire, soit de décrire le visiteur de musée. La mise en place de ce type d'approche, dans laquelle il s'agit de tester des hypothèses, nécessite des protocoles le plus souvent lourds. Plusieurs études ont été nécessaires, seule la dernière de ces études est présentée ici ${ }^{1}$.

\section{Le questionnaire}

L'étude présentée s'appuie sur un questionnaire en trois parties: 1-questions sur l'image de soi, 2-questions sur l'image du visiteur des musées des beaux-arts, 3-questions sur quelques pratiques muséales, avec un relevé d'informations sur certaines données sociodémographiques.

Chaque partie du questionnaire est glissée dans une enveloppe séparée. Les personnes enquêtées ont été invitées à respecter scrupuleusement l'ordre indiqué sur les enveloppes et à n'ouvrir l'enveloppe suivante qu'après avoir répondu au contenu de l'enveloppe précédente.

- La première enveloppe contient une liste de 24 adjectifs $^{2}$ permettant aux personnes enquêtées de se décrire ${ }^{3}$ :

- 12 décrivent le visiteur de musée,

- 12 renvoient à des traits de personnalité extraits de l'Adjective Check List (ACL) de Gough 4 : sans rapport avec l'image du visiteur, ils sont introduits ici comme « distracteurs ».

\begin{tabular}{|cccc|}
\hline \multicolumn{4}{c|}{ Les adjectifs } \\
dits «pertinents » (12) \\
$\begin{array}{c}\text { décrivant } \\
\text { le visiteur de musée }\end{array}$ & \multicolumn{2}{c|}{$\begin{array}{c}\text { ne décrivant pas } \\
\text { particulièrement } \\
\text { le visiteur de musée }\end{array}$} \\
& & Actif & Gai \\
Artiste & Passionné & Direct & Généreux \\
Attentif & Rêveur & Dynamique & Organisé \\
Calme & Sensible & Efficace & Persévérant \\
Cultivé & Tolérant & Exigeant & Perspicace \\
Curieux & Ouvert & Précis & Rationnel \\
Imaginatif & Patient & & \\
\hline
\end{tabular}

- La deuxième enveloppe contient la même liste à partir de laquelle les personnes enquêtées doivent décrire le visiteur de musée des beaux-arts ${ }^{5}$.

- La troisième enveloppe contient une série de questions auxquelles les personnes enquêtées sont invitées à répondre concernant leur profil sociodémographique et leurs pratiques des musées (fréquentation, attitude à l'égard des visites de musée, niveau de compétence auto-évaluée).

La fréquentation des musées a ainsi été évaluée pour la totalité des personnes interrogées, à partir de trois modalités de réponse («pas de visite », « une ou deux visites », «trois visites et plus " au cours de l'année).

\section{L'échantillon}

La constitution de l'échantillon a visé à une certaine hétérogénéité du point de vue de l'âge des personnes et de leur formation. Compte tenu de la nature des travaux à effectuer et de leur durée, ce sont surtout des étudiants qui ont été choisis, bien que des personnes qui ne l'étaient pas aient été contactées afin d'avoir des individus plus âgés offrant un rapport à l'art et aux musées différent de celui des étudiants. Par ailleurs, le fait qu'il y ait des étudiants dans l'échantillon a permis de contrôler la variable formation en art (incluant les étudiants de l'École du Louvre) et de travailler sur son effet. Il a été également possible de travailler sur l'effet du type de cursus suivi en distinguant les filières scientifiques des autres filières (variable peu prise en compte dans les études).

L'échantillon, composé de 381 individus, comprend des étudiants de Paris 10 et de Nancy 2, des étudiants de l'École du Louvre et des personnes n'appartenant pas au contexte universitaire.

Un peu plus des deux tiers des enquêtés sont des femmes. L'âge médian est de 22 ans : dans l'ensemble les personnes sélectionnées sont jeunes, mais $25 \%$ d'entre elles ont 33 ans et plus.

L'échantillon est composé majoritairement d'étudiants de premier cycle (38\%) et de master (33\%).

Plus d'un quart (26\%) d'entre eux sont issus d'un milieu d'ouvriers ou d'employés, $34 \%$ d'un milieu de cadres moyens ou supérieurs.

1. Hana Gottesdiener, Jean-Christophe Vilatte et Pierre Vrignaud, Pratiques culturelles et image de soi : le cas de la fréquentation des musées, Paris, Deps, Ministère de la Culture et de la Communication, 2007

2. Seuls des adjectifs positifs sont proposés pour trois raisons, les deux premières ayant trait à l'image des visiteurs de musée, la dernière à l'image de soi : 1 . la préétude montrait que très peu d'adjectifs négatifs étaient produits par les enquêtés pour décrire les visiteurs de musée; 2 . la fréquence d'occurrence de qualificatifs négatifs pour décrire le visiteur de musée ne semble pas liée avec la pratique muséale; 3 . enfin il n'était pas souhaitable de proposer des adjectifs qui puissent donner une image de soi négative, la comparaison d'une telle image avec celle du visiteur risquant d'être l'objet de biais difficiles à contrôler et à évaluer.

3. La consigne était la suivante : «Pour chacune des caractéristiques suivantes nous vous demandons de vous situer - en mettant une croix - sur la ligne allant de « Ne me décrit pas du tout » à « Me décrit tout à fait », en face de chacun des adjectifs ». Les 24 adjectifs ont été sélectionnés à partir d'une pré-étude sur les représentations du visiteur; différentes approches méthodologiques ont été testées au cours de cette étude préliminaire: production libre d'adjectifs qualifiant le visiteur de musée et adjectifs à choisir parmi une liste tirée de l'AcL de Gough. Cette préétude a permis de distinguer des adjectifs caractéristiques du visiteur de musée d'art des adjectifs qui ne le sont pas.

4. L'ACL est un outil polyvalent qui permet d'évaluer la personnalité et la perception des tendances psychologiques. II se compose de 300 adjectifs qui se répartissent en cinq catégories (chaleur sociale, conformisme, efficacité, maturité et extraversion) qui se regroupent en 37 échelles. Il est utilisé le plus souvent en autodescription.

5. La consigne était la suivante: « Maintenant, nous vous demandons, pour chacune des caractéristiques suivantes de décrire le visiteur de musée des beauxarts (Louvre, Orsay...) en le situant, par une croix, sur la ligne allant de « Ne le décrit pas du tout » à « Le décrit tout à fait », pour chaque adjectif. La même codification a été utilisée que pour l'image de soi. 
Dans un peu plus d'un tiers des cas, les mères des personnes interrogées ont fait des études supérieures et dans $44 \%$ des cas, elles ont le niveau baccalauréat. La répartition des niveaux d'études est très proche en ce qui concerne les pères.

Les limites de l'échantillon ainsi posées, les résultats n'en proposent pas moins des pistes de réflexion qui renouvellent l'appréhension des rapports des individus aux musées.

\section{Mode de calcul de la distance image de soi-image du visiteur}

Pour chacun des adjectifs présents sur la liste, les personnes interrogées doivent se positionner sur une échelle de 1 à 5 , allant de 1 «ne me décrit pas du tout » à 5 «me décrit tout à fait » (ou décrire le visiteur sur cette même échelle).

La distance est ensuite calculée dans le sens image de soi, image du visiteur:
- une distance positive entre les deux images signifie que l'individu considère que l'adjectif le décrit mieux qu'il ne décrit le visiteur de musée des beaux-arts. Plus la distance est importante, plus la personne s'attribue à un niveau élevé le qualificatif ;

- inversement, une distance négative entre les deux images signifie que l'individu considère que l'adjectif proposé le qualifie moins bien qu'il ne qualifie le visiteur de musée des beaux-arts. Cette distance est d'autant plus forte que l'adjectif semble éloigné de lui-même.

Dans les études précédentes, on avait fait l'hypothèse que l'adoption d'un comportement était d'autant plus probable que la distance entre images était nulle. Ici, nous avons mis en évidence qu'un écart positif était favorable au déclenchement du comportement, et qu'inversement, un écart négatif lui était défavorable. Prendre la distance non signée (valeur absolue ou distance quadratique) aboutissait à confondre les écarts positifs et négatifs et donc à ne plus trouver d'effets. 


\section{RÉSUMÉ}

Cette recherche se propose de tester l'hypothèse selon laquelle la fréquentation des musées d'art peut être prédite à partir de la distance entre l'image de soi et l'image que la personne a des visiteurs. L'outil de mesure construit a été utilisé auprès de 381 personnes. L'échantillon composé surtout d'étudiants présente cependant une certaine hétérogénéité du point de vue de l'âge et de la formation. Les résultats montrent en particulier que si la distance entre les images est positive dans le cas de ceux qui visitent les musées, elle est négative dans le cas contraire. Ceux qui n'ont pas de pratique de visite ont tendance à se sous-estimer par rapport à l'image qu'ils ont du visiteur de musée. Il est alors nécessaire de s'interroger sur un accompagnement qui permettrait de réduire cette distance négative.

\section{ABSTRACT}

This research is intended to test the assumption that art gallery attendance can be predicted from the disparity between self-image and a person's idea of gallery-goers. The measuring tool devised by us was applied to a sample of 381 persons. Although the sample is made up primarily of students, it displays a certain variety in terms of age and education. The findings show in particular that, while the disparity is positive in the case of gallery-goers, it is negative in the opposite case. The persons who do not go to galleries tend to downgrade themselves in comparison with the image they have of those who do. This illustrates a need to investigate what sort of guidance might succeed in narrowing the negative disparity. 